\title{
Wind Turbine Gearbox Temperature Monitor Based On CITDMFPA-WNN Baoyi Wang ${ }^{a}$, Wuchao Liu ${ }^{b}$, Shaomin Zhang ${ }^{c}$ \\ ${ }^{1}$ School of Control and Computer Engineering, North China Electric Power University, Baoding, 071003, China \\ aemail:wangbaoyi@126.com, ${ }^{\mathrm{b} e m a i l: 519299582 @ q q . c o m, ~}{ }^{\mathrm{c} e m a i l}$ :zhangshaomin@126.com
}

Keywords: gearbox; temperature monitoring; flower pollination algorithm; wavelet neural network

\begin{abstract}
In the gearbox temperature monitoring, in order to improve the predictive accuracy of gearbox temperature, we presents a model that improved flower pollination algorithm optimizes wavelet neural network (CITDMFPA-WNN) used in gearbox temperature prediction. Use the model to predict the temperature of the gearbox, and then get the gearbox condition by means of the analysis of residual temperature, so as to achieve the goal of gearbox online temperature monitoring. By introducing chaos sequence and t distribution variation, flower pollination algorithm (FPA) has better optimization ability. Improved FPA is used to optimize the uncertain parameters of wavelet neural network that improves the training speed and precision of wavelet neural network. Experiments show that the forecast precision of CITDMFPA-WNN is better than that of IPSO-WNN and WNN, and we can find the abnormal temperature of gearbox and achieve the goal of real-time online monitoring.
\end{abstract}

\section{Introduction}

Gearbox, one of the key components of wind turbine, because of the bad running environment, has a high incidence failure, which causes the high cost of the wind farm operation and maintenance ${ }^{[1]}$. According to statistics, the gearbox faults account for about $10 \%$ of the total, but their downtime is more than $50 \%$ of the total downtime, so temperature monitoring of the gear box, timely detection of fault symptoms, determine the reasonable maintenance program are of great significance to reduce maintenance costs and improve the reliability of the unit. Haiyu Mao, Guoqi Liu, et $\mathrm{al}^{[2]}$ use principal component analysis to remove irrelevant attributes of the operation data of wind farm and then train the wavelet neural network to get the fault offset vector groups, so as to achieve the purpose of fault diagnosis. P. Bangalore and L. B. Tjernberg ${ }^{[3]}$ use a lot of data in the SCADA system, including alarm and warning information to train the artificial neural network for the fault prediction of wind turbine gearbox bearing, and the experiment indicates that the bearing fault can be found in advance. However, the [2, 3] do not take into account the structure of the neural network. With the multi dimension data, the convergence rate of the neural network is slow, and it is easy to fall into the local optimum. Wenxian Yang, Tavner P J, Crabtree C J, et al ${ }^{[4]}$ set up the hardware experimental platform of gearbox and generator, use the wavelet analysis method to analyze the vibration signal of high speed acquisition, but the result has a larger difference with the actual running state of the gearbox. In reality, the acquisition speed of the vibration signal is often difficult to meet the needs of high frequency vibration analysis. In this paper, the method of temperature trend analysis is used to monitor the condition of the gearbox. Firstly, an improved flower pollination algorithm is proposed to optimize the wavelet neural network method to set up the temperature model of the gearbox, and the temperature of the gearbox is predicted by the model. Then get the gearbox condition by means of the analysis of residual temperature, so as to achieve the goal of gearbox online temperature monitoring. Based on the standard of flower pollination algorithm, the chaotic sequence and variation of $t$ distribution are introduced to form a kind of improved flower pollination algorithm. The improved flower pollination algorithm is used for wavelet neural network parameter optimization, so as to improve the training speed and prediction accuracy of wavelet neural network. 


\section{Analysis Of Factors Affecting The Temperature Of The Gearbox Of The Wind Turbine}

In recent years, the SCADA system has been widely used in various wind farms. The main function of the system is to collect and record the state data of each component or subsystem of the wind turbine. Select SCADA system monitoring data of a wind farm in Inner Mongolia in 2013, whose acquisition time interval is $10 \mathrm{~min}$, including recording time, wind speed, active power, reactive power, rotor speed, ambient temperature, gearbox input shaft temperature, gearbox output shaft temperature and a total of 47 parameters. The rated power of the wind turbine is $1.5 \mathrm{MW}$, cut into the wind speed is $3 \mathrm{~m} / \mathrm{s}$, the rated wind speed is $11 \mathrm{~m} / \mathrm{s}$, the cut out wind speed is $25 \mathrm{~m} / \mathrm{s}$. Taking oil temperature of the gear for example, we use the correlation coefficient method to obtain the main factors affecting the gearbox oil temperature. Calculate the correlation coefficient between the 46 variables in the SCADA system including the oil temperature of the upper time and the temperature of the gearbox oil. Some calculation results are shown in table 1.

Table 1 The correlation coefficient of gearbox oil temperature and other variables

\begin{tabular}{cccc}
\hline wind speed & power of wind turbine & generator speed & environment temperature \\
\hline 0.6166 & 0.7002 & 0.8136 & 0.1881 \\
\hline $\begin{array}{c}\text { gearbox input shaft } \\
\text { temperature }\end{array}$ & $\begin{array}{c}\text { gearbox output shaft } \\
\text { temperature }\end{array}$ & $\begin{array}{c}\text { the oil temperature } \\
\text { of the upper time }\end{array}$ & \\
\hline 0.9584 & 0.9595 & 0.9758 & \\
\hline
\end{tabular}

Select the correlation coefficients greater than 0.6 variables as the main factors affecting the gearbox oil temperature, thus the result is the wind speed (S), power of wind turbine $(\mathrm{P})$, gearbox speed $(V)$, the oil temperature of the upper time $\left(T_{0}\right)$, gear box input shaft temperature $\left(T_{1}\right)$, the gearbox output shaft temperature $\left(\mathrm{T}_{2}\right)$.

\section{Design Of Gearbox Temperature Prediction Model}

Design Of Temperature Prediction Model Based On Wavelet Neural Network. Wavelet neural network (WNN) not only has the local time-frequency characteristics of wavelet transform characteristic of the large-scale data but also possesses parallel processing and self-learning ability of neural networks, so it has faster convergence speed and stronger convergence ability. In this paper, the 3 layer wavelet neural network is used to predict the oil temperature of the gearbox, and the structure is shown in Figure 1.

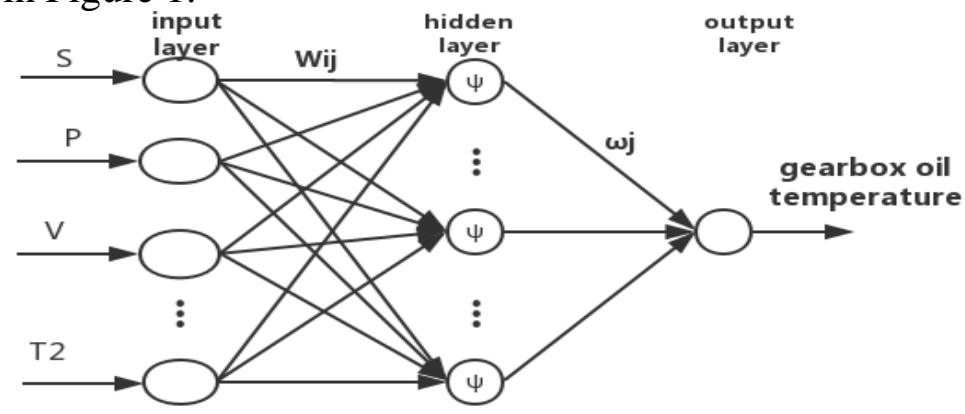

Fig.1. Structure of wavelet neural network

Input layer: the number of nodes in the input layer is related to the input variables. Input variables directly affect the accuracy of the prediction results. Therefore, we put the wind speed (S), power of wind turbine $(\mathrm{P})$, gearbox speed $(\mathrm{V})$, the oil temperature of the upper time $\left(\mathrm{T}_{0}\right)$, gear box input shaft temperature $\left(T_{1}\right)$, the gearbox output shaft temperature $\left(T_{2}\right)$ as input variables, namely $\mathrm{X}=\left[\mathrm{X}_{1}, \mathrm{X}_{2}, \mathrm{X}_{3}, \mathrm{X}_{4}, \mathrm{X}_{5}, \mathrm{X}_{6}\right]=\left[\mathrm{S}, \mathrm{P}, \mathrm{V}, \mathrm{T}_{0}, \mathrm{~T}_{1}, \mathrm{~T}_{2}\right]$, and the number of nodes and input variables the number of the corresponding. The input of the $\mathrm{j}$ node of the wavelet element is as follows.

$$
h_{j}=\sum_{i=1}^{m} w_{i j} X_{i}, j=1,2, \ldots, n
$$

In the formula, $\mathrm{w}_{\mathrm{ij}}$ is the connection weights between the input layer node $\mathrm{i}$ and the hidden layer node $\mathrm{j}, \mathrm{X}_{\mathrm{i}}$ is the $\mathrm{i}$ input variable, $\mathrm{m}$ is the number of the input layer nodes and $\mathrm{n}$ is the number of hidden layer nodes.

Hidden layer: compared with Morlet wavelet and DOG wavelet, error of Marr wavelet decreased 
most quickly, and Marr wavelet has the least number of iterations to complete the wavelet neural network, so that we use Marr wavelet function as the hidden layer wavelet neuron ${ }^{[5]}$. Marr wavelet is defined as follows.

$$
\phi(t)=\frac{2}{3} \pi^{1 / 4}\left(1-t^{2}\right) e^{-t^{2} / 2},-\infty<t<+\infty
$$

The output of the first $\mathrm{j}$ neuron in the hidden layer is

$$
z_{j}=\phi\left(\frac{h_{j}-b_{j}}{a_{j}}\right), j=1,2, \ldots, n
$$

In the formula, $a_{j}$ and $b_{j}$ are the expansion factor and translation factor of the node of the hidden layer in the first $\mathrm{j}$.

Output layer: this layer corresponds are the predicted results, that is, the gearbox oil temperature. The number of output layer nodes is 1 . Network output is as follows.

$$
y=1 /\left(1+\exp \left(-\sum_{j=1}^{n} \omega_{j} z_{j}\right)\right)
$$

In the formula, $\omega_{\mathrm{j}}$ is the connection weights between the hidden layer node $\mathrm{j}$ and the output layer.

In the known structure of wavelet neural network, whether the wavelet neural network is suitable to solve the problem lies in the selection of parameters in the network or not, it depends on the optimization of the network training algorithm $\zeta=(w, a, b, \omega)$. Traditional wavelet neural network training algorithms are mostly BP algorithm. However, BP algorithm has the disadvantages of slow convergence and easy to fall into local optimum. So it is very important to find a suitable training algorithm of wavelet neural network ${ }^{[6]}$.

Parameter Optimization Of Gearbox Temperature Prediction Model .Flower pollination algorithm (FPA) has a few parameters and is easy to adjust, which not only has the strong global optimization capability of the cuckoo algorithm but also has the ability to balance the global search and local search of the bat algorithm ${ }^{[7]}$. Therefore, we use FPA as the training algorithm of wavelet neural network. However, there is a slow convergence rate and it is easy to fall into local optimum in the FPA iterative optimization process. So we make improvements from the following two aspects.

1. Chaotic sequence initialization

The chaotic sequence is ergodic, stochastic, regular and so on. Therefore, we use chaotic sequence to initialize the population of FPA. Logistic mapping and cubic mapping are the most commonly used chaotic models, but the cubic map has better uniformity than Logistic maps, so we chooses cubic map ${ }^{[8]}$.Its expression is as follows.

$$
y(n+1)=4 y(n)^{3}-3 y(n),-1 \leq y(n) \leq 1, n=0,1,2, \ldots
$$

2. $T$ distribution variation strategy

T distribution was published by William Gore Jose in 1980, which can be controlled by changing the parameter $n$ of the degree of freedom to control the shape of the curve. In order to increase the population diversity and improve the accuracy, we use the t distribution variation strategy ${ }^{[9]}$. In addition to optimal in vitro, variant strategies like formula (6).

$$
X_{i}{ }^{\prime}=X_{i}+X_{i}^{*} q^{*} t(k)
$$

Here, $X_{i}$ ' is the first $i$ pollen individual after variation, $X_{i}$ is the first i pollen individual before mutation, $\mathrm{t}(\mathrm{k})$ is the current iteration times, $\mathrm{k}$ is the parameter of the $\mathrm{t}$ distribution, $\mathrm{q}$ is the control factor, which is expressed as the formula (7).

$$
q=1-\frac{k}{D-1}, k=0,1, \ldots, D-1
$$

In the formula, $\mathrm{D}$ is the maximum number of iterations of the algorithm, and $\mathrm{k}$ is the current iteration number.

For the current optimal individual, its solution is closest to the optimal solution, so it should be less variable and the variation strategy is like the formula (8).

$$
X_{\text {best }}{ }^{\prime}=X_{\text {best }}+X_{\text {best }} * q * N(0,1)
$$


In view of the shortcomings of slow convergence and easy to fall into local optimum, we present a new flower pollination algorithm based on $t$ distribution variation and chaotic sequence initialization(CITDMFPA), which is described as follows:

Step 1: Initialize parameters: including the number of population, the population individual dimension, the transition probability, the maximum number of iterations, search space, etc;

Step 2: Initialize the population by chaos sequence, calculate the fitness value of each individual, and obtain the optimal fitness value and its corresponding solution;

Step 3: Randomly generate the parameter rand, if rand $<\mathrm{p}$, then do global search, otherwise do local search.

Step 4: Calculate the fitness value in Step 3. If the new fitness value is better than the original, replace the current solution and the current fitness value with the new explanation and corresponding fitness value, otherwise keep the current solution and the current fitness value.

Step 5: Do t mutation operation following the formula (6) or the formula (8).Calculate the fitness value of variant individuals.

Step 6: Judge whether to meet the end conditions, if meeting, stop the iteration and output optimal solution, otherwise, turn to Step 3.

\section{Design Of Gearbox Temperature Prediction Algorithm}

After the WNN structure is determined WNN will be trained, and the average mean square error (MAPE) is chosen as the precision function.

$$
f_{\text {mape }}=\frac{1}{n} \sum_{i=1}^{n}\left|\frac{y_{i}-y_{i}{ }^{\prime}}{y_{i}}\right|
$$

Here, $\mathrm{n}$ is the sample number, $\mathrm{y}_{\mathrm{i}}$ is the real value, and $\mathrm{y}_{\mathrm{i}}{ }^{\prime}$ is the predicted value.

We use CITDMFPA to optimize the parameter of wavelet neural network, and then use the trained WNN to predict the temperature of the key parts of the gearbox. Gearbox temperature prediction algorithm(CITDMFPA -WNN) is as follows:

Step 1: Initialize CITDMFPA parameters.

Step 2: Input training sample. Use the individual value of CITDMFPA as the parameter value of WNN and output prediction results by prediction model and calculate the fitness value according to formula (9).

Step 3: According to CITDMFPA, update each individual. Bring the updated individual into the wavelet neural network, calculate and update the individual fitness value.

Step 4: Judge whether to meet the end conditions, if meeting, stop the iteration and output the overall optimal solution and obtain the optimal parameters. Otherwise, turn to Step 3.

Step 5: According to the predicted sample, use the optimal parameters to predict the temperature of the gearbox.

\section{Experiment And Numerical Analysis}

Experimental Data Set Preprocessing .Select SCADA system monitoring data of a wind farm in Inner Mongolia in 2013 and 2014, and the acquisition time interval is 10min. Select the normal operation data from April 2013 to March 2014 as the training samples and the training output is the gearbox oil temperature. The input data is normalized by the formula (10).

$$
X_{i}^{\prime}=\frac{X_{i}-X_{\min }}{X_{\max }-X_{\min }}
$$

In the formula, $\mathrm{X}_{\min }$ and $\mathrm{X}_{\max }$ are the minimum and maximum values of the original data. $\mathrm{X}_{\mathrm{i}}$ is the original data and $\mathrm{X}_{\mathrm{i}}$ ' is the normalized data.

Test Results And Analysis Of CITDFPA-WNN Model. We select 100 normal operating data in May 4, 2014 as a test sample, and select IPSO algorithm in the literature [6] optimization WNN algorithm, WNN algorithm, CITDMFPA-WNN algorithm for comparison. In order to guarantee the objectivity of the experimental data, the algorithm performs 10 times and the average value is the 
final result. The predicted results are shown in Figure 1 and MAPE is shown in Table 2.

As can be seen from Table 2 and Figure 1, CITDMFPA-WNN algorithm is superior to the WNN algorithm which is not optimized, and is better than the IPSO-WNN algorithm.

Table 2 MAPE comparison of each algorithm

\begin{tabular}{cccc}
\hline Prediction Algorithm & CITDMFPA-WNN & IPSO-WNN & WNN \\
\hline MAPE & 0.0240 & 0.0318 & 0.0421 \\
\hline
\end{tabular}

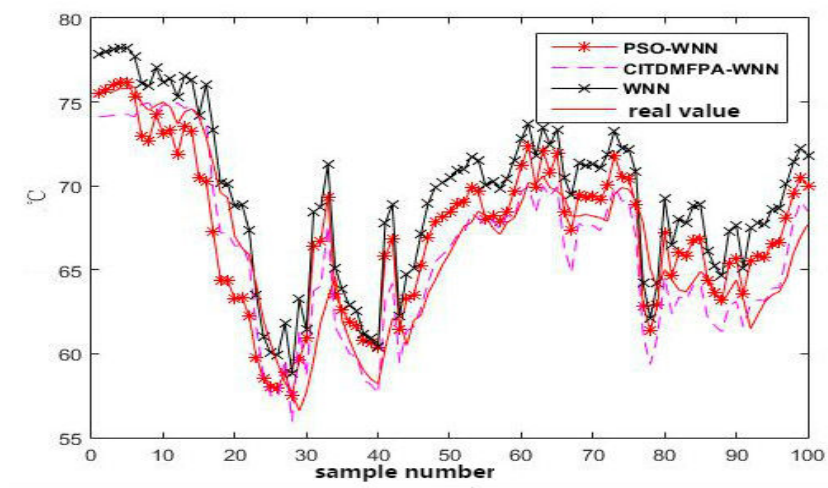

Fig. 1 The true value and predicted value of gearbox oil temperature

Verification Of Gearbox Temperature Monitoring Method.We select the normal operation data (2000 samples) of 15 consecutive days in April 2014 for gearbox oil temperature prediction. The residual error of the gearbox oil temperature is recorded $a s u_{c}$, and the formula is as formula (11).

$u_{c}=y-y^{\prime}$

In the formula, $y$ is the gearbox oil temperature measured and $y^{\prime}$ is the gearbox oil temperature prediction value.

In this paper, the method of sliding window statistical method is used to analyze the temperature residual error to determine the threshold value of the mean and standard deviation of the gearbox. The results are shown in Figure 2. The threshold of gearbox oil temperature under the normal state is as follows.

$\left\{\begin{array}{l}E= \pm k_{E} E_{\text {max }} \\ S=k_{S} S_{\text {max }}\end{array}\right.$

In the formula, $E_{\max }$ is the maximum value of the mean value of the residual series. $S_{\max }$ is the maximum value of standard deviation. $\mathrm{k}_{\mathrm{e}}$ and $\mathrm{k}_{\mathrm{s}}$ are real number and can be determined by the field operation personnel according to experience.

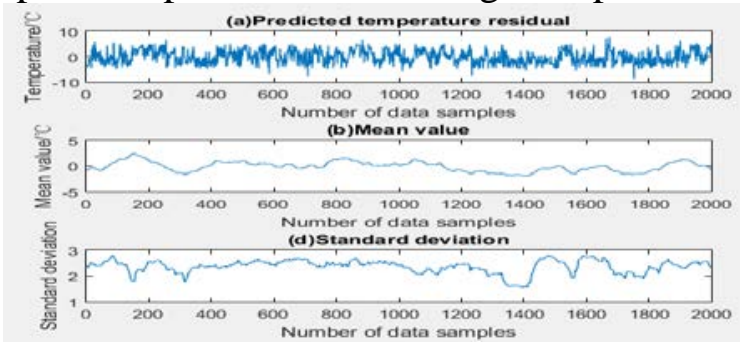

Fig. 2 Gearbox oil temperature residual, mean value and standard deviation

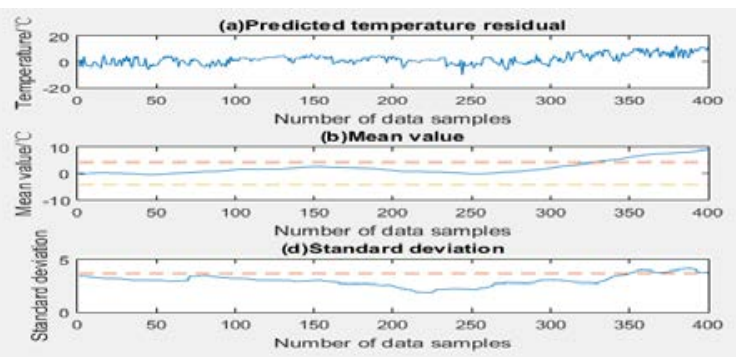

Fig. 3 Gearbox oil temperature monitoring

In the Figure 2, $\mathrm{E}_{\max }=2.15, \mathrm{~S}_{\max }=2.81$. So we take $\mathrm{k}_{\mathrm{E}}=2, \mathrm{k}_{\mathrm{S}}=1.3$, then the gearbox oil hydraulic failure threshold value is: $E= \pm 4.30, S=3.65$.In order to verify the validity of the model to monitor the temperature fault of the gearbox oil, artificial simulation of gear box oil high temperature fault is done. Select May 3, 20140 o'clock to 6 o'clock, all of the 576 sets of historical data, and start man-made step cumulative temperature offset $0.02^{\circ} \mathrm{C}$ from 350th. Oil temperature and the residual prediction results of gearbox of fault simulation are shown in Figure 3.

As can be seen from Figure 3, the residual mean value is more than normal threshold at 325 . So the distance from the initial point 350 is $325+100-350=75$ and here the width of the sliding window 
is 100. Therefore, in the 425th place, we can find out the abnormal condition of the gearbox oil. At this point, the gearbox oil temperature deviation is $75^{*} 0.02=1.4^{\circ} \mathrm{C}$. It can be seen that when the gear box because of the fault and the temperature rise, the method can be found in time and send out the alarm, realize the gear box online temperature monitoring.

\section{Summary}

In this paper, the WNN is used to set up the temperature model of gearbox with the process variables which are closely related to the temperature of the gearbox, and the improved FPA is used to optimize the WNN. According to the distribution characteristic of the residual error of the model, we uses the sliding window to calculate the variation trend of the residual mean and standard deviation in real time in real time, and set the threshold comparison to get the current operational status of the gearbox. Experiments show that the forecast precision of CITDMFPA-WNN is better than that of IPSO-WNN and WNN, and we can find the abnormal temperature of gearbox and achieve the goal of real-time online monitoring.

\section{Acknowledgement}

In this paper, the research was sponsored by the National Natural Science Foundation of China (Project No. 61300040) and Scientific Research Project of Hebei Province (Project No. Z2012077).

\section{References}

[1] Li Hui, Hu Yaogang, Li Yang, Yang Dong, Liang Yuanyuan, Ouyang Haili, LAN Yongsen. Overview of condition monitoring and fault diagnosis for grid-connected high-power wind turbine unit [J]. Electric power automation equipment, 2016,01:6-16.

[2] Liu Guoqi, Mao Haiyu, Zhu Yongfeng, Pu Baoming, et al. Fault Diagnosis of Wind Turbines Based on Wavelet Neural Network [J]. Small and micro computer system, 2015,07:1504-1508.

[3] P. Bangalore and L. B. Tjernberg, An Artificial Neural Network Approach for Early Fault Detection of Gearbox Bearings[J]. IEEE Transactions on Smart Grid, March 2015,vol. 6, no. 2, pp. 980-987.

[4] Yang Wenxian,Tavner P J, Crabtree C J, et al. Cost-effective condition monitoring for wind turbines [J] . IEEE Transactions on Industrial Electronics,2010, 57(1):263-271.

[5] Shu Jian, Guo Kai, Liu Qun, Liu Lin-Lan, Research of Connectivity Parameters in Opportunistic Sensor Networks[J/OL], Chinese Journal of Computers,2015, Vo1.38: Online Publishing No.155.

[6] Cheng Shengfeng, Yang Lu, Cheng Xiaohua. Application of wavelet neural network with improved particle swarm optimization algorithm in power transformer fault diagnosis [J]. Power system protection and control, 2014,19:37-42.

[7] Xiao Huihui, wan chang Xuan, Duan. Improved novel metaheuristie flower pollination algorithm [J]. Computer application research, 2016,01:126-131.

[8] Zhou Yan, Liu Peiyu, Wang Qianlong, Zhao Jing. Chaos particle swarm optimization algorithm based on adaptive inertia weight [J]. Journal of Shandong University (SCIENCE EDITION), 2012,03:27-32.

[9] Du Xiaoxin, Zhang Jianfei. Sun Ming.Artificial glowworm swarm optimization algorithm based on adaptive t distribution mixed mutation[J].Computer application,2013,07:1922-1925+1972. 